\title{
The effect of wet-cupping therapy (hijama) in modulating autoimmune activity of Hashimoto's thyroiditis
}

\author{
A pilot controlled study
}

Amal M. Obeid, MD, Faiza A. Qari, MD, Soad K. Aljaouni, MD, Sawsan Rohaiem, MD, Abmed A. Elsayed, MD, Maha M. Alsayyad, MD, Ezzuddin A. Okmi, MD.

\begin{abstract}
الأهداف : بحث تأثير الحجامة على الالتهاب المناعي للغدة الدرقية في حالات

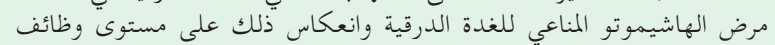
هرمونات الغدة في حالات الهاشيموتو للغدة المنتظمة وانعاع.

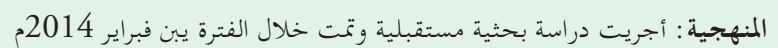

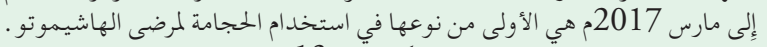

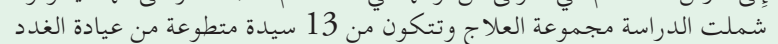

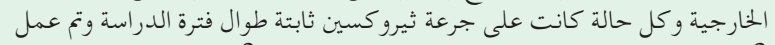

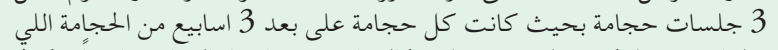

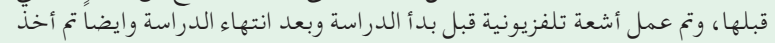

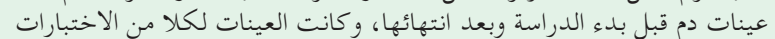

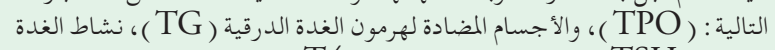

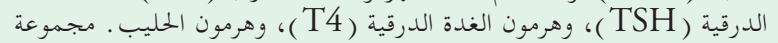

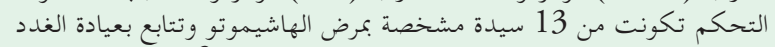

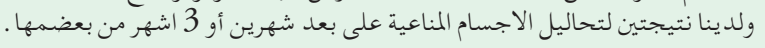

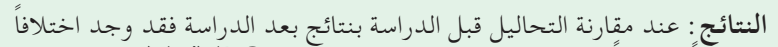

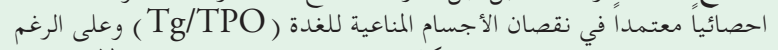

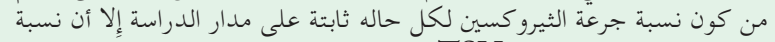

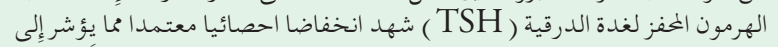

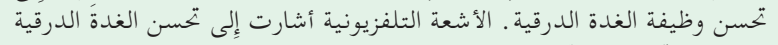

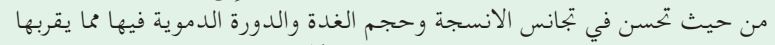

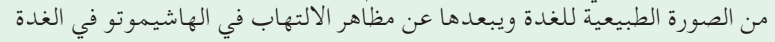
المصابة بالهاشيموتو . ميعن.

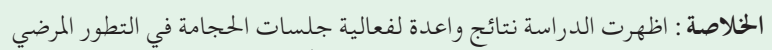

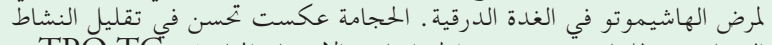

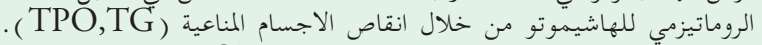

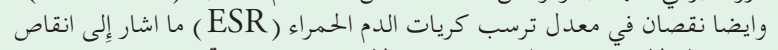

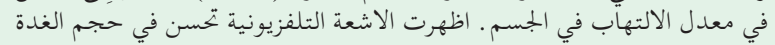

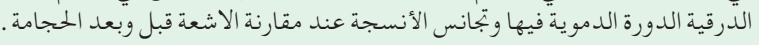

Objectives: To investigate the possible effects of wetcupping therapy (hijama) on autoimmune activity and thyroid hormonal profiles of Hashimoto's disease (HD) patients.

Methods: A prospective pilot controlled trial was carried out among adult females following HD at the Endocrinology Clinic of King Abdulaziz University Hospital, Jeddah, Saudi Arabia, from February 2014 until March 2017. A total of 13 patients (intervention group) underwent 3 hijama sessions at 3-week interval with a 6-month follow-up. Blood tests were carried out for both pre- and post-hijama, including: thyroid peroxidase antibody (TPO), thyroglobulin antibody (TG), thyroid stimulating hormone (TSH), thyroxine-4 (T4), and prolactin. Control group included 13 randomly selected HD patients following usual care with 2 consecutive measurements of TPO and TG at 2-3 months interval.

Results: Changes in anti-TPO and anti-TG showed a decrease in hijama group versus an increase in control group, and intervention group comparison was statistically significant. On a fixed thyroxine supplement for each patient, significant decrease in levels of anti-TPO, anti-TG, TSH, prolactin, and erythrocyte sedi-mentation rate (ESR) occurred.

Conclusion: These findings are presumptive to the efficacy of hijama on the pathogenesis of HD in modulating the immune inflammatory process demonstrated by the reduction in ESR, TPO, and TSH levels on a fixed thyroxin supplementation dose, along with patientreported clinical improvement and positive changes in ultrasound including regaining of gland physiological size, echogenicity, and vascularity.

Keywords: hijama, cupping, Hashimoto's thyroiditis, thyroiditis, thyroid gland

Saudi Med J 2022; Vol. 43 (1): 45-52

doi: 10.15537/smj.2022.43.1.20210755

From the Department of YAJ chair for Prophetic medical applications (Obeid, Alsayyad), Cupping Therapy Clinics; from the Department of Hematology (Aljaouni), Yousef Abdulatif Jameel Scientific Chair of Prophetic Medicine Application, from the Department of Medicine (Qari); from the Department of Physiology (Rohaiem); From the Faculty of Medicine (Alsayyad), King Abdulaziz University, Jeddah, from the Department of Communicable Diseases Prevention and Control (Okmi), Public Health Authority, Riyadh, Kingdom of Saudi Arabia, and from the Department of Radiology (Elsayed), The Royal Wolverhampton Trust, New Cross Hospital, London, United Kingdom.

Received 29th September 2021. Accepted 23rd November 2021.

Address correspondence and reprint request to: Dr. Ezzuddin A. Okmi, Communicable Diseases Prevention and Control Department, Public Health Authority, Riyadh, Kingdom of Saudi Arabia. E-mail:dr.e_okmi@hotmail.com

ORCID ID: https://orcid.org/0000-0003-2634-3143 
$\mathrm{H}^{2}$ ashimoto's thyroiditis, or Hashimoto's disease (HD) is an organ-specific autoimmune disease characterized by abnormal infiltration of lymphocytes in thyroid follicles and the production of anti-thyroid auto-antibodies by B-cells. The thyroid antibodies are mainly against thyroid peroxidase (TPO) and thyroglobulin (TG) enzymes. However, TPO is the most sensitive indicator for HD. ${ }^{1}$ This humoral autoimmunity is combined with cellular mechanisms that involve T-cell cytotoxicity. ${ }^{2-5}$ Patients with HD are managed by long-life oral supplementation of thyroid hormone, L-thyroxine, with monitoring of serum thyroid function level. ${ }^{6}$

Hashimoto's thyroiditis is frequently associated with other autoimmune diseases such as vitiligo, poly glandular autoimmune disorders, coeliac disease, and type I diabetes, especially in children. ${ }^{7,8}$ There are several factors associated with HD, such as susceptibility genes, iodine intake, drugs, stress, and infections. These factors are likely to be interacting, causing immunological alteration and the release of thyroid specific antigens, thus, breaking immunological self-tolerance and stimulating autoreactive lymphocytes. ${ }^{9,10}$

Despite the growing literature on the therapeutic uses of wet-cupping therapy (hijama in Arabic) in various pathology, controversy remains regarding its efficacy. This controversy is mainly due to the limited quality of studies resulting in inconclusive clinical evidences. ${ }^{11,12}$ Among the limitations of hijama studies is the difficulty to build up a double-blind controlled model to provide a strong evidence of the observed therapeutic effects. Furthermore, explaining the mechanisms of hijama may be challenging, as this would require substantial resources considering the lack of funding for such researches.

In autoimmune diseases, it was suggested that hijama improved symptoms of the disease by clearing blood and interstitial fluid from the noxious substances such as auto-antibodies, immune complexes, pro-inflammatory mediators, cytokines, oxidants, and soluble cytokine receptors. ${ }^{13-16}$ This immune-modulatory effect was described in rheumatoid arthritis, where hijama induced significant reduction in rheumatoid factor and soluble interleukin-2 receptors along with clinical improvement including pain and arthritis. ${ }^{17}$ Several authors have studied the efficacy of hijama in various inflammatory, autoimmune or infectious conditions

Disclosure. Authors have no conflict of interests, and the work was not supported or funded by any drug company. such as asthma, psoriasis, facial paralysis, herpes zoster, cervical spondylitis, and acne. ${ }^{16,18-20}$

In a prospect to extend the therapeutic utility of hijama in autoimmune diseases, we carried out this pilot study investigating the possible effects of hijama on autoimmune markers and thyroid hormonal profiles of patients with HD treated with conventional therapy.

Methods. A prospective controlled clinical trial was carried out among adult female patients following $\mathrm{HD}$ at the Endocrinology outpatient clinic of King Abdulaziz University Hospital (KAUH), Jeddah, Saudi Arabia. Patients aged between 19-65 years receiving L-thyroxin and having high antithyroid antibodies levels by reference to normality ranges used in our laboratory facility were included. Exclusion criteria were patients below 19 years or above 65 years, those with normal antithyroid antibodies level, pregnant women, as well as those with bleeding disorders. A pre-trial face-to-face interview was carried out with patients from interventional group, during which the study procedure was explained, signed, and informed consents were obtained. As for the control group, patients were randomly selected from the hospital electronic system among all patients following $\mathrm{HD}$ in the Endocrinology clinic and verifying the same eligibility criteria. Additionally, patients from control group should have at least 2 consecutive measurements of TPO and TG antibodies, the primary outcome, at approximately $2-3$ months interval. The study protocol was approved by the Institutional Review Board of KAUH, Jeddah, KSA. (Reference no. 167-15).

The interventional group included 13 adult women, who accepted to undergo a 3-month hijama therapy as an adjuvant to a well carried out, conventional treatment for HD. The intervention consisted of 3 hijama sessions at a 3-week interval for a total intervention duration of 6 weeks. Hijama sessions were scheduled at the begining of the trial and were carried out in the Prophetic Medicine Clinics, KAUH.

Locations of the cups consisted of the anatomical locations of main organs that are assumed to be involved in the pathological process, which are thyroid gland, submandibular lymph node if demonstrated to be enlarged in ultrasound (US), sternal angle (targeting bone marrow and atrophied thymus gland) for possible immune modulation effect. Bone marrow and thymus represent the 2 principal sites for immune tolerance where auto reactivity of B-cells and T-cells are modulated. ${ }^{16}$

The hijama session included 5 steps: i) disinfection using alcohol; ii) first cup suction, prior to incision using single use special hijama cups and hijama suction 
gun; iii) minimal skin incision using a size 15 sterile surgical blades to make approximately 10 incisions per cup location, each incision being approximately $2-3 \mathrm{~mm}$ long and $1 \mathrm{~mm}$ depth; iv) cup suction for 30 seconds to 1 minute; v) end of suction and dressing application.

In the interventional group clinical, laboratory, and US assessments were carried out twice, at baseline prior to the first hijama session, and 3 weeks after the end of the third hijama session (approximately 9-10 weeks interval between baseline and outcome assessment). Blood markers included anti-TPO and anti-TG antibodies, which were the primary outcomes of this study, and were completed by TSH, T4, T3, prolactin, and erythrocyte sedi-mentation rate (ESR). Clinical assessment included the presence and severity of the 5 common clinical signs of hypothyroidism including constipation, period regularity, energy level, concentration ability, and sleep quality. Ultrasound assessment included cervical lymph nodes and thyroid gland size, vascularity, and homogeneity.

Statistical analysis. Statistical Package for the Social Sciences, version 21 for Windows (IBM Corp., Armonk, NY, USA) was used. Inter-group analysis compared mean TPO and TG antibodies levels in intervention versus control groups, both at baseline and 2 months as well as the mean baseline-to-outcome change, using both T-test (parametric) and Mann-Whitney-U test (nonparametric). Inter-group analysis was carried out to compare baseline and outcome values of TPO, TG, $\mathrm{TSH}, \mathrm{T} 4$, ESR, and prolactin levels in intervention group using both parametric (paired t-test) and nonparametric (Wilcoxon signed rank) tests. Results are presented as mean \pm standard deviation (SD). A $p$-value of $<0.05$ was considered to reject the null hypothesis.

Results. In baseline, hijama group had lower levels of anti-TPO (mean difference: $-148.93 \mathrm{IU} / \mathrm{ml}$ ) and higher levels of anti-TG (mean difference: $114.97 \mathrm{IU} / \mathrm{ml}$ ); and a statistical significance was found only for anti-TPO in nonparametric $(p=0.047)$ but not in parametric tests $(p=0.217)$. Baseline-to-3-month change in anti-TPO showed a mean of $60.47 \mathrm{IU} / \mathrm{ml}$ decreased in hijama group, versus a mean of $98.95 \mathrm{IU} / \mathrm{ml}$ increased in control group; and the result was statistically significant using parametric test $(p=0.030)$ but not in nonparametric test $(p=0.067)$. Additionally, anti-TG decreased by $229.06 \mathrm{IU} / \mathrm{ml}$ in hijama group while it increased by $28.39 \mathrm{IU} / \mathrm{ml}$ in usual care group; this difference was statistically significant using both parametric $(p=0.017)$ and nonparametric $(p<0.001)$ tests (Table 1$)$.

Baseline-to-3 months nonparametric paired analysis of serum markers in hijama group showed significant decrease in the following; prolactin $(p=0.028)$, TPO $(p=0.003)$, and TG $(p=0.013)$ levels. Parametric tests confirmed the statistical significance of this reduction of prolactin $(p=0.045)$ and TG $(p=0.019)$, but not of TPO $(p=0.153)$. Additionally, both TSH and ESR decreased. However, this was only significant in parametric tests for TSH and only in nonparametric tests for ESR (Table 2). Figure 1 presents the baseline and post-hijama values of TPO, TG, TSH, and T4 levels in the intervention group; showing decrease in values of the majority of patients.

Baseline-to-3-months paired analysis of TPO and TG was also carried out in usual care (control) group, showing increasing trend in TPO (mean change: $98.95 \mathrm{IU} / \mathrm{ml} ; p=0.263)$ and statistically significant increase in TG (mean change: $28.39 \mathrm{IU} / \mathrm{ml} ; p=0.018$; Table 3).

Table 4 summarizes self-assessed improvements (in percentage) in 8 clinical signs, among patients in hijama group. The majority of patients reported 50-100\% improvement in the following symptoms: constipation, period regularity, energy level, concentration ability, and sleep quality.

Table 1 - Effect of a 3-wet-cupping therapy (hijama) sessions at 3 weeks interval on TPO and TG levels in Hashimoto's thyroiditis by comparison to usual care.

\begin{tabular}{|c|c|c|c|c|c|c|c|}
\hline \multirow[t]{2}{*}{ Markers/time } & \multicolumn{2}{|c|}{ Hijama group $(n=13)$} & \multicolumn{2}{|c|}{ Control group $(n=13)$} & \multirow[t]{2}{*}{ Mean difference } & \multicolumn{2}{|c|}{$P$-value } \\
\hline & Mean \pm SD & Range & Mean \pm SD & Range & & Par. & Npar. \\
\hline \multicolumn{8}{|l|}{ TPO } \\
\hline $\begin{array}{l}\text { Baseline } \\
\text { Outcome } \\
\text { Change }\end{array}$ & $\begin{array}{c}153.00 \pm 264.55 \\
86.20 \pm 185.63 \\
-60.47 \pm 129.61\end{array}$ & $\begin{array}{c}0.15-813.58 \\
0.01-662.86 \\
-\end{array}$ & $\begin{array}{c}301.93 \pm 304.82 \\
397.76 \pm 396.23 \\
98.95 \pm 173.95\end{array}$ & $\begin{array}{c}7.14-1000 \\
7.42-1000 \\
-\end{array}$ & $\begin{array}{l}-148.93 \\
-311.57 \\
-159.43\end{array}$ & $\begin{array}{l}0.219 \\
0.026^{*} \\
0.030^{*}\end{array}$ & $\begin{array}{c}0.047^{*} \\
0.015^{*} \\
0.067\end{array}$ \\
\hline \multicolumn{8}{|l|}{$T G$} \\
\hline $\begin{array}{l}\text { Baseline } \\
\text { Outcome } \\
\text { Change }\end{array}$ & $\begin{array}{c}314.06 \pm 349.75 \\
84.99 \pm 106.58 \\
-229.06 \pm 273.38\end{array}$ & $\begin{array}{c}3.85-1078.00 \\
1.83-338.08 \\
-\end{array}$ & $\begin{array}{c}199.09 \pm 365.57 \\
227.48 \pm 349.53 \\
28.39 \pm 27.27\end{array}$ & $\begin{array}{c}4.30-444.30 \\
4.69-486.50 \\
-\end{array}$ & $\begin{array}{l}114.97 \\
-142.86 \\
257.45\end{array}$ & $\begin{array}{l}0.492 \\
0.216 \\
0.017^{*}\end{array}$ & $\begin{array}{c}0.177 \\
0.492 \\
<0.001^{*}\end{array}$ \\
\hline
\end{tabular}

TPO: thyroid peroxidase, TG: thyroglobulin, SD: standard deviation, Par: parametric (independent t-test), Npar: nonparametric (Mann Whitney-U test), "statistically significant result (2-tailed $p$-value of $<0.05)$ 
Table 2 - Evolution of thyroiditis serum markers in hijama group (paired analysis, n=9).

\begin{tabular}{lccccc}
\hline Parameters & \multicolumn{2}{c}{ Before hijama } & After hijama & Mean change & \multicolumn{2}{c}{$P$-value } \\
& \multicolumn{2}{c}{ Mean \pm SD } & & Par. & Npar. \\
\hline Prolactin & $331.58 \pm 129.43$ & $211.73 \pm 62.18$ & -119.85 & $0.045^{*}$ & $0.028^{*}$ \\
TPO & $150.84 \pm 252.35$ & $94.74 \pm 184.49$ & -56.10 & 0.147 & $0.003^{*}$ \\
TG & $197.14 \pm 338.59$ & $89.16 \pm 102.64$ & -207.97 & $0.022^{*}$ & $0.013^{*}$ \\
ESR & $112.80 \pm 197.64$ & $73.60 \pm 131.64$ & -39.20 & 0.256 & $0.043^{*}$ \\
T4 & $15.08 \pm 1.99$ & $15.11 \pm 3.09$ & 0.03 & 0.963 & 0.342 \\
TSH & $5.47 \pm 5.64$ & $3.48 \pm 3.73$ & -1.99 & $0.008^{*}$ & 0.195 \\
\hline
\end{tabular}

SD: standard deviation, Par: parametric (paired t-test), Npar: nonparametric (Wilcoxon signed rank test), TPO: thyroid peroxidase, TG: thyroglobulin, ESR: erythrocyte sedi-mentation rate, T4: thyroxine 4, TSH: thyroid stimulating hormone, "statistically significant result $(2$-tailed $p$-value of $<0.05)$

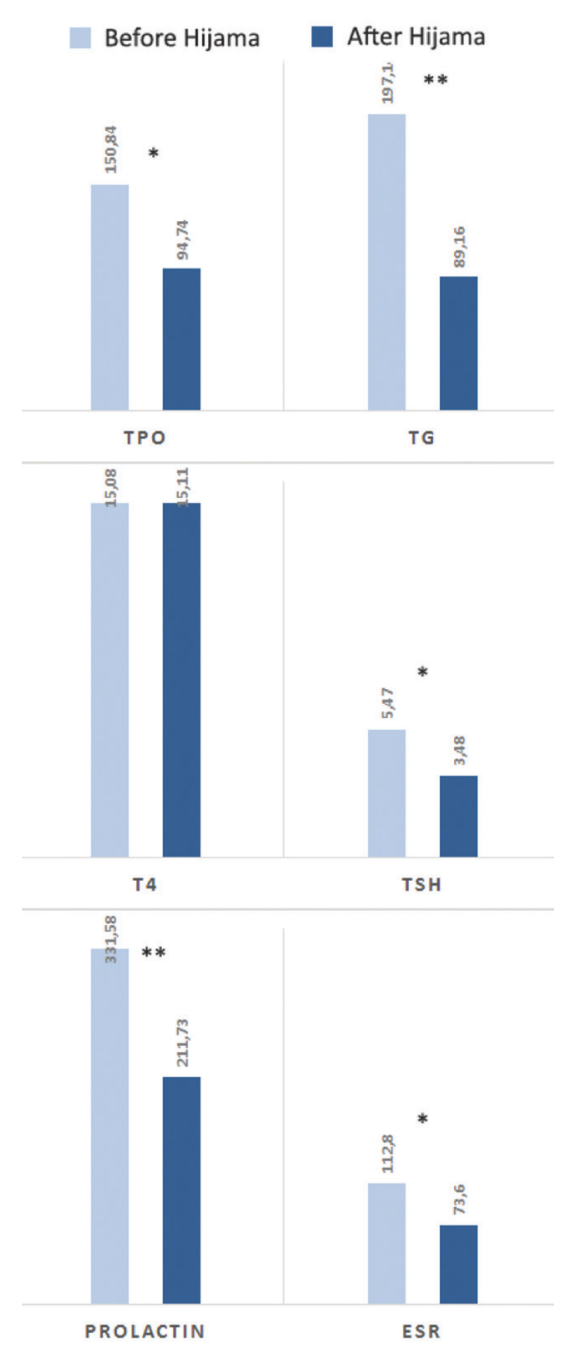

Figure 1 - Change in thyroiditis serum markers from baseline to 3 weeks post Hijama in the interventional group. TPO: thyroid peroxidase, TG: thyroglobulin, T4: thyroxine 4, TSH: thyroid stimulating hormone, ESR: erythrocyte sedi-mentation rate, "statistical significance in parametric or nonparametric, "statistical significance in both parametric and nonparametric tests
Regarding US changes with hijama therapy, 4 patients had enlarged thyroid size at baseline, 2 of them had size reduction after hijama. A total of 9 patients had abnormal baseline thyroid vascularization (7 hyper-vascularization and 2 hypo-vascularization), 7 of them showed improved vascularization after hijama. A total of 9 had enlarged lymph nodes before hijama, 5 of them had normal lymph nodes appearance after hijama. Figure 2 illustrates the remarkable US changes in selected patients.

Discussion. The application of wet cupping therapy (hijama) in this study may constitute a hope for the patients suffering from HD. The idea of this trial was based on the hypothesis of the observed immune modulatory effects of hijama on some autoimmune conditions that may also be effective in HD, which would be of a great value since HD is considered the most common autoimmune thyroid disease. ${ }^{21,22}$ Furthermore, the development of thyroid cancer was found to be related to the prolonged exposure of high anti-thyroid auto antibodies level and associated pro-inflammatory environment. ${ }^{23-25}$ The possibility of slowing down the autoimmune process might prevent further destruction of the gland and improve its function, which represents a good hope for HD patients. This could also be of an advantage in preventing further development of thyroid cancer, which is an interesting point to explore. The treatment option for HD is basically thyroid replacement therapy. ${ }^{26}$ If hijama was found to be beneficial in enhancing thyroid physiological function and slowing its autoimmune destruction, it could be of value to explore the worthiness of adding it to the current therapeutic protocol of HD management.

Although not conclusive, findings from this pilot study are presumptive for the efficacy of 3 hijama sessions in modulating autoimmune activity in HD. This was demonstrated by the statistically significant 
Table 3 - Evolution of TPO and TG in usual care group (paired analysis, n=9).

\begin{tabular}{|c|c|c|c|c|c|}
\hline \multirow[t]{2}{*}{ Parameters } & Baseline & At 3 months & \multirow[t]{2}{*}{ Mean change } & \multicolumn{2}{|c|}{$P$-value } \\
\hline & \multicolumn{2}{|c|}{ Mean $\pm S D$} & & Par. & Npar. \\
\hline TPO & $298.81 \pm 338.23$ & $397.76 \pm 396.23$ & 98.95 & 0.126 & 0.263 \\
\hline TG & $199.09 \pm 356.67$ & $227.48 \pm 349.53$ & 28.39 & $0.022^{*}$ & $0.018^{*}$ \\
\hline
\end{tabular}

TPO: thyroid peroxidase, TG: thyroglobulin, SD: standard deviation, Par: parametric (paired t-test), Npar: nonparametric (Wilcoxon signed rank test), "statistically significant result $(2$-tailed $p$-value of $<0.05)$

Table 4 - Clinical changes after 3 hijama sessions among patients with Hashimoto’s disease.

\begin{tabular}{|c|c|c|c|c|c|c|c|c|}
\hline Patients & Hair & voice & Mood & Constipation & $\begin{array}{r}\text { Period regularity } \\
\text { Precentage }(\%\end{array}$ & Energy level & Concentration ability & Sleep disturbance \\
\hline 1 & - & - & 50 & 60 & 50 & 90 & 50 & 70 \\
\hline 2 & - & - & - & - & - & 50 & - & - \\
\hline 3 & - & - & - & 100 & 100 & 100 & 100 & 100 \\
\hline 4 & 50 & - & - & - & - & 50 & - & - \\
\hline 5 & - & - & - & - & 100 & 50 & - & - \\
\hline 6 & 50 & 50 & - & 50 & 50 & 50 & 50 & 50 \\
\hline 7 & 50 & 50 & - & 50 & 50 & 50 & 50 & 50 \\
\hline 8 & - & - & - & - & - & 50 & 50 & 50 \\
\hline 9 & - & - & 50 & 50 & 50 & 50 & 50 & 50 \\
\hline 10 & 50 & - & - & - & 50 & - & - & 50 \\
\hline 11 & - & - & - & 100 & 100 & - & - & 50 \\
\hline \multicolumn{9}{|l|}{12} \\
\hline 13 & - & - & 50 & 60 & 50 & 90 & 50 & 70 \\
\hline
\end{tabular}

reduction in anti-thyroid auto antibodies, both through inter-group and inter-patients comparative analyses. Additionally, a reduction in inflammatory marker, ESR, was observed. Literature shows that the levels of circulating anti-TPO and anti-TG auto antibodies were found to be positively correlated with inflammation and hypothyroidism, and very high levels are predictive for disease aggressiveness and thyroid destruction. ${ }^{22}$

Furthermore, treated patients showed improvement in all aspects, hormonally, morphologically, and clinically. Hormonally, TSH showed significant reduction in spite of a fixed thyroid dose with all patients throughout the study. This is supportive of an increased thyroid function. Prolactin, which is associated with hypothyroid function was significantly reduced. ${ }^{1}$ A negative feedback effect from improved thyroid function on pituitary gland is interesting to explore. Ultrasound demonstrated morphological improvement in thyroid gland and associated cervical lymph nodes. Patients' symptoms were also clinically improved in spite of the short follow-up time ( 3 months). All findings are empowering the idea of decreased thyroid inflammation, prevention of the inflammatory destruction of thyroid gland, improved thyroid tissue status, and enhanced thyroid function.
An interesting point to explore in future studies is that the decreasing trend in TPO was related to fibrosis and destruction of the thyroid gland due to prolonged HD effect. Thus, ruling out fibrosis would further support that the decreased TPO is related to the hijama effect.

Careful scrutiny of pre-hijama TPO levels in the intervention group showed increasing trend among 4/13 patients and slowly increasing trend in 11 others. This is a suggestive of the efficacy of hijama not only in stopping but also in reversing the autoimmune process of HD patients. However, more adequate study design is warranted to confirm this observation.

Ultrasound is a key investigation in HD. The reduced echogenicity of thyroid gland is a diagnostic criterion, besides the elevation of anti-TPO and anti-TG levels. ${ }^{26}$ Furthermore, there is a positive correlation between the degree of hypo-echogenicity and anti-thyroid auto antibodies levels, as well as the level of TSH. ${ }^{27}$ Other sonographic findings in HD included: heterogeneity, hyper-vascularity, hyper-echogenicity, and eventual presence of goiter or micro nodules. ${ }^{28}$ Ultrasound may also show linear structures corresponding to fibrosis. Finally, in case of nodules or other images suspicious for malignancy, echo-guided biopsy can be carried out. ${ }^{29}$ In 
Hijama normalized enlarged thyroid gland (epithelium diameter)

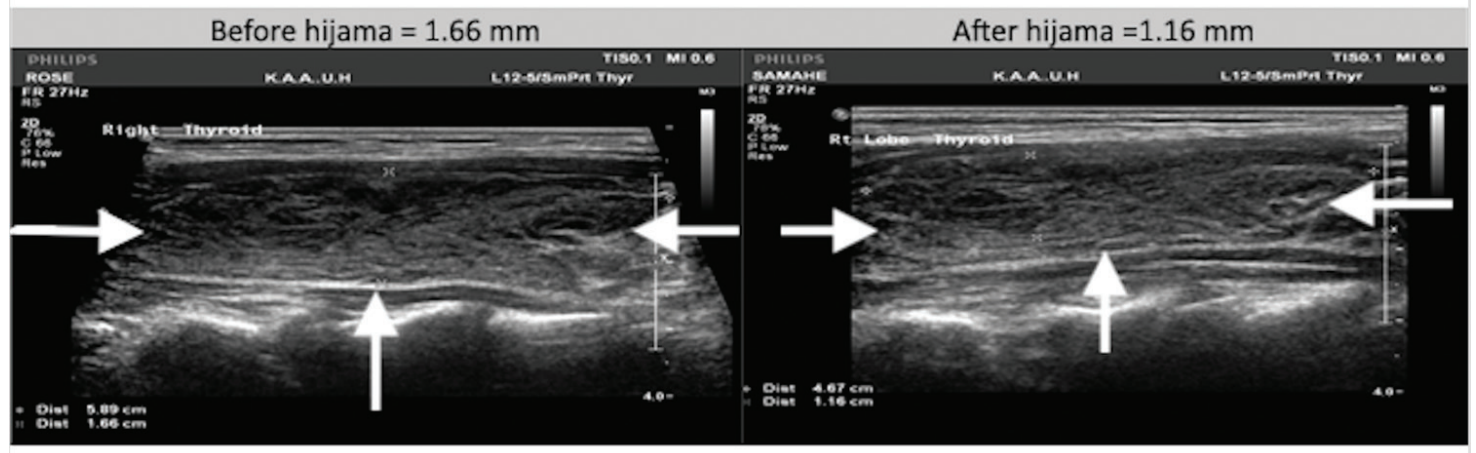

Hijama effect on thyroid hypervacularity

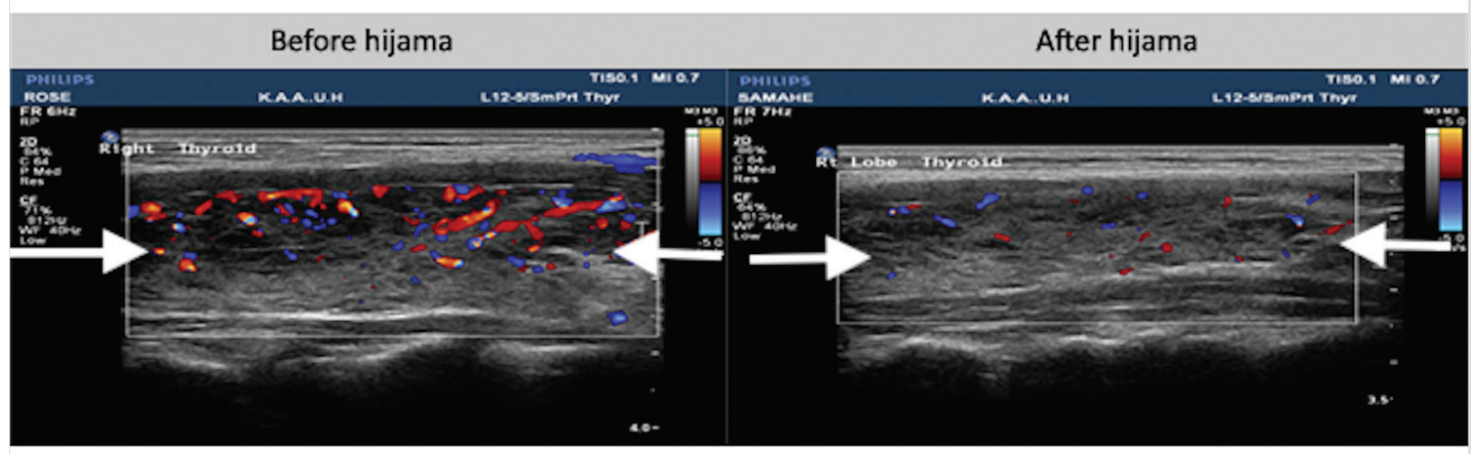

Hijama effect on thyroid hypovacularity

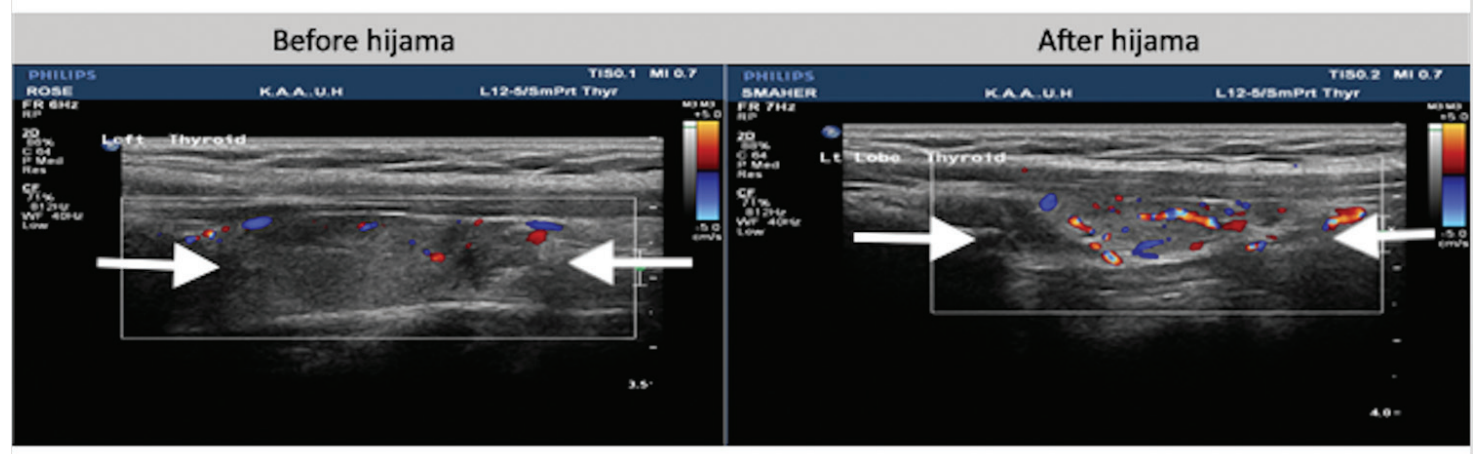

Hijama effect on thyroid Heterogeneity

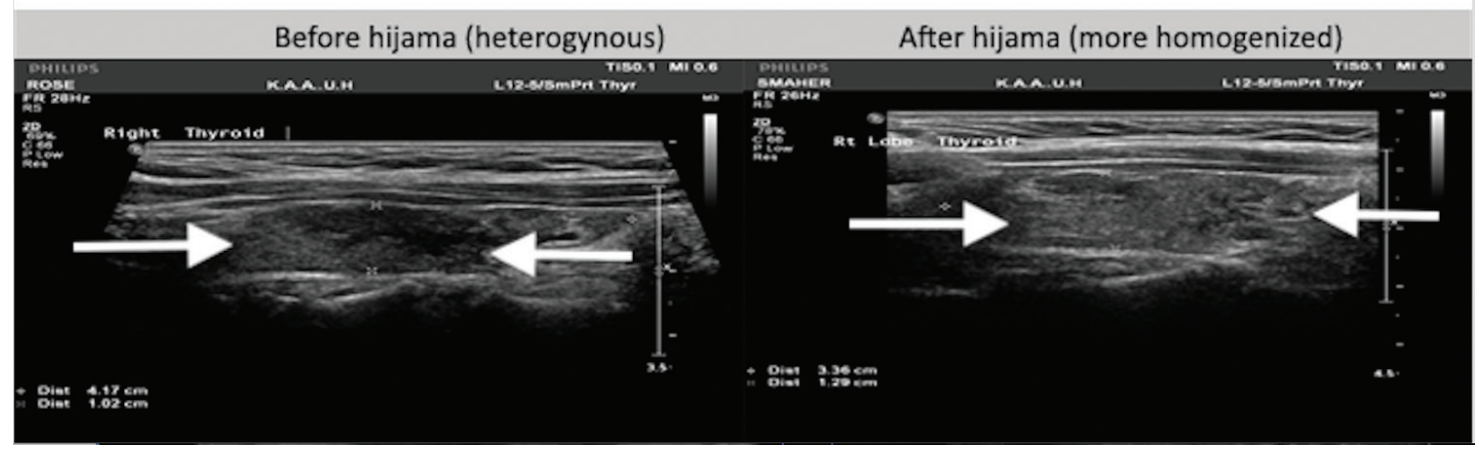

Figure 2 - Morphological changes in thyroid ultrasound assessments in hijama group. 
this study, all US parameters were promising in terms of improving thyroiditis degree and enhancing thyroid vitality, and function.

In sum, Findings from this pilot study support the efficacy of hijama in reversing the autoimmune and inflammatory processes of HD, thereby, normalizing the histological and physiological status of the gland and enhancing its function. To explain these potential effects, several possible mechanisms may be involved. First, the physical suction exerted may result in cleansing the regional tissues from inflammatory mediators such as histamine, inflammatory cytokines, and autoantibodies, which are filtered out of capillaries, intracellular, and interstitial spaces to the subcutaneous area, and to the hijama cup via the skin incision. Although the present study did not analyze the cupped blood to confirm this hypothesis, this was supported by previous studies reporting higher concentration of oxidative and inflammatory mediators in wet-cupping blood. ${ }^{30}$ Other data from literature suggested that hijama helps restoring humoral immunity and tissue homeostasis by clearing up serum and interstitial fluid from noxious substances that sustain inflammation. ${ }^{13-16}$

Some studies suggested that toxic metals can increase the risk of developing thyroid diseases. ${ }^{31,32}$ Moreover, mercury can play a role in the pathogenesis of autoimmune thyroiditis by triggering autoimmune reactions and oxidative damage. ${ }^{32}$ Therefore, the level of higher heavy metal in cupping blood than venous blood can explain the mechanism of wet-cupping therapy in improving patients with thyroid diseases such as HD by removing heavy metals. ${ }^{33,34}$ The second theory is mainly related to the enhancement of tissue vasculature and blood flow. The physical suction induces hyperemia with eventual dilatation of blood vessels, which enhances blood circulation, on one hand, and accelerates tissue clearance from inflammatory mediators, on the other hand. The 2 mechanisms would result in restoring a good homeostasis and vitality of the gland by providing an optimum vasculature and environmental status. Furthermore, the decreased prolactin level would contribute in these effects is an interesting point to explore. Previous data demonstrated that increased prolactin level was associated with hypothyroid levels. ${ }^{1}$ The mechanism underlying this decrease in prolactin should be further explored and may involve a negative feedback effect of restored thyroid hormones on the pituitary gland, thus decreasing both TSH and prolactin. Further research is warranted to explore all possible mechanisms underlying these effects.

Study limitations. This study was limited by the small sample size and different data collection method for the intervention and control group. In addition, there were several missing clinical, biochemical and imaging data, which prevented the controlling of baseline parameters such as the duration, stage of the disease, and other confounders. Despite these limitations, findings from this study are promising and call for further trials with adequate designs.

In conclusion, this pilot controlled study supports the hypothesis of hijama efficacy in modulating autoimmune and inflammatory activity of HD and enhancing thyroid function. This was demonstrated by the reduction in ESR, TPO, and TSH levels on a fixed thyroxin supplementation dose, along with patientreported clinical improvement and positive changes in US including regaining of gland physiological size, echogenicity, and vascularity. Such effects highlight that hijama is a promising effective and safe alternative or adjuvant therapy in HD, which represents a big hope for millions of HD patients. Further prospective controlled trials with adequate design and sample size are warranted to confirm the efficacy of hijama in HD and to provide more insight into the mechanisms of such effects.

Acknowledgment. The authors gratefully acknowledge WAX Clinical Research Skills Limited for English language editing.

\section{References}

1. Avasthi K, Kaur J, Gupta S, Narang PA. Hyperprolactinemia and its correlation with hypothyroidism in infertile women. $J$ Obs Gynecol India 2006; 56: 68-71.

2. Caturegli P, Kimura H, Rocchi R, Rose NR. Autoimmune thyroid diseases. Curr Opin Rheumatol 2007; 19: 44-48.

3. Ajjan RA, Weetman AP. The pathogenesis of Hashimoto's thyroiditis: further developments in our understanding. Horm Metab Res 2015; 47: 702-710.

4. Wiersinga WM. Clinical relevance of environmental factors in the pathogenesis of autoimmune thyroid disease. Endocrinol Metab (Seoul) 2016; 31: 213-222.

5. Fröhlich E, Wahl R. Thyroid autoimmunity: role of antithyroid Antibodies in thyroid and extra-thyroidal diseases. Front Immunol 2017; 8: 521.

6. Canaris GJ, Manowitz NR, Mayor G, Ridgway EC. The Colorado thyroid disease prevalence study. Arch Intern Med 2000; 160: 526-534.

7. Lorini R, Gastaldi R, Traggiai C, Perucchin PP. Hashimoto's thyroiditis. Pediatr Endocrinol Rev 2003; 1: 205-211.

8. Radetti G. Clinical aspects of Hashimoto's thyroiditis. Endocr Dev 2014; 26: 158-170.

9. Chistiakov DA. Immunogenetics of Hashimoto's thyroiditis. $J$ Autoimmune Dis 2005; 2: 1.

10. Zaletel K, Gaberšček S. Hashimoto's thyroiditis: from genes to the disease. Curr Genomics 2011; 12: 576-588.

11. AlBedah A, Khalil M, Elolemy A, Elsubai I, Khalil A. Hijama (cupping): a review of the evidence. Focus Altern Complement Ther 2011; 16: 12-16. 
12. Sajid MI. Hijama therapy (wet cupping) - its potential use to complement British healthcare in practice, understanding, evidence and regulation. Complement Ther Clin Pract 2016; 23: 9-13.

13. El Sayed SM, Mahmoud HS, Nabo MMH. Methods of wet cupping therapy (Al-hijamah): in light of modern medicine and prophetic medicine. Altern Integr Med 2013: 1-16.

14. El Sayed SM, Al-quliti AS, Mahmoud HS, Baghdadi H, Maria RA, Nabo MMH, et al. Therapeutic benefits of Al-hijamah: in light of modern medicine and prophetic medicine. Am J Med Biol Res 2014; 2: 46-71.

15. Tagil SM, Celik HT, Ciftci S, Kazanci FH, Arslan M, Erdamar $\mathrm{N}$, et al. Wet-cupping removes oxidants and decreases oxidative stress. Complement Ther Med 2014; 22: 1032-1036.

16. Baghdadi H, Abdel-Aziz N, Ahmed NS, Mahmoud HS, Barghash A, Nasrat A, et al. Ameliorating role exerted by Al-hijamah in autoimmune diseases: effect on serum autoantibodies and inflammatory mediators. Int J Health Sci (Qassim) 2015; 9: 207-232.

17. Ahmed SM, Madbouly NH, Maklad SS, Abu-Shady EA. Immunomodulatory effects of blood letting cupping therapy in patients with rheumatoid arthritis. Egypt J Immunol 2005; 12 : $39-51$.

18. Cao H, Han M, Li X, Dong S, Shang Y, Wang Q, et al. Clinical research evidence of cupping therapy in China: a systematic literature review. BMC Complement Altern Med 2010; 10: 70.

19. Cao H, Li X, Liu J. An updated review of the efficacy of cupping therapy. PLoS One 2012; 7: e31793.

20. Malik IA, Akhter S, Kamal MA. Treatment of psoriasis by using hijamah: a case report. Saudi J Biol Sci 2015; 22: 117-121.

21. Brown RS. Autoimmune thyroid disease: unlocking a complex puzzle. Curr Opin Pediatr 2009; 21: 523-528.

22. Pyzik A, Grywalska E, Matyjaszek-Matuszek B, Roliński J. Immune disorders in Hashimoto's thyroiditis: what do we know so far? J Immunol Res 2015; 2015: 979167.

23. Kim ES, Lim DJ, Baek KH, Lee JM, Kim MK, Kwon HS, et al. Thyroglobulin antibody is associated with increased cancer risk in thyroid nodules. Thyroid 2010; 20: 885-891.

24. Muzza M, Degl'Innocenti D, Colombo C, Perrino M, Ravasi E, Rossi S, et al. The tight relationship between papillary thyroid cancer, autoimmunity and inflammation: clinical and molecular studies. Clin Endocrinol (Oxf) 2010; 72: 702-708.
25. McLeod DS, Cooper DS, Ladenson PW, Ain KB, Brierley JD, Fein HG, et al. Prognosis of differentiated thyroid cancer in relation to serum thyrotropin and thyroglobulin antibody status at time of diagnosis. Thyroid 2014; 24: 35-42.

26. Caturegli P, De Remigis A, Rose NR. Hashimoto thyroiditis: clinical and diagnostic criteria. Autoimmun Rev 2014; 13: 391-397.

27. Schiemann U, Avenhaus W, Konturek JW, Gellner R, Hengst K, Gross M. Relationship of clinical features and laboratory parameters to thyroid echogenicity measured by standardized grey scale ultrasonography in patients with Hashimoto's thyroiditis. Med Sci Monit 2003; 9: MT13-MT17.

28. Koprowski R, Zieleźnik W, Wróbel Z, Małyszek J, Stępień B, Wójcik W. Assessment of significance of features acquired from thyroid ultrasonograms in Hashimoto's disease. Biomed Eng Online 2012; 11: 48.

29. Gul K, Dirikoc A, Kiyak G, Ersoy PE, Ugras NS, Ersoy R, et al. The association between thyroid carcinoma and Hashimoto's thyroiditis: the ultrasonographic and histopathologic characteristics of malignant nodules. Thyroid 2010; 20: 873-878.

30. Ahmed SM, Madbouly NH, Maklad SS, Abu-Shady EA. Immunomodulatory effects of blood letting cupping therapy in patients with rheumatoid arthritis. Egypt J Immunol 2005; 12 : 39-51.

31. Rezaei M, Javadmoosavi SY, Mansouri B, Azadi NA, Mehrpour $\mathrm{O}$, Nakhaee $\mathrm{S}$. Thyroid dysfunction: how concentration of toxic and essential elements contribute to risk of hypothyroidism, hyperthyroidism, and thyroid cancer. Environ Sci Pollut Res Int 2019; 26: 35787-35796.

32. Pamphlett R, Doble PA, Bishop DP. Mercury in the human thyroid gland: potential implications for thyroid cancer, autoimmune thyroiditis, and hypothyroidism. PLoS One 2021; 16: e0246748.

33. Umar NK, Tursunbadalov S, Surgun S, Welcome MO, Dane S. The effects of wet cupping therapy on the blood levels of some heavy metals: a pilot study. J Acupunct Meridian Stud 2018; 11: 375-379.

34. Gok S, Kazanci FH, Erdamar H, Gokgoz N, Hartiningsih S, Dane $S$. Is it possible to remove heavy metals from the body by wet cupping therapy (al-hijamah)? India J Tradit Knowle 2016; 15: 700-704. 